\title{
Air-Stable Secondary Phosphine Oxides for Nickel-Catalyzed Cross-Couplings of Aryl Ethers by $\mathrm{C}-\mathrm{O}$ Activation
}

\author{
Debasish Ghorai ${ }^{\mathrm{a}, \mathrm{b}}$ \\ Joachim Loup ${ }^{a}$ \\ Giuseppe Zanoni ${ }^{\mathrm{b}}$ \\ Lutz Ackermann*a (1) \\ a Institut für Organische und Biomolekulare Chemie, Georg-August-Universität, \\ Tammannstraße 2, 37077 Göttingen, Germany \\ Lutz.Ackermann@chemie.uni-goettingen.de \\ ${ }^{b}$ Department of Chemistry, University of Pavia, Viale Taramelli 10, 27100 Pavia, \\ Italy \\ Published as part of the 30 Years SYNLETT - Pearl Anniversary Issue
}

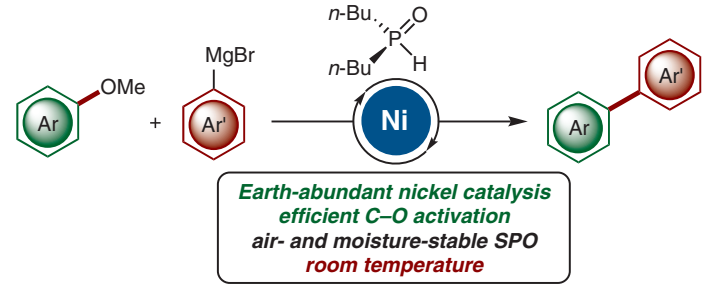

Received: 02.12.2018

Accepted after revision: 06.01.2019

Published online: 15.01 .2019

DOI: 10.1055/s-0037-1611663; Art ID: st-2018-b0782-I

License terms: cc)

Abstract Air- and moisture-stable secondary phosphine oxides (SPOs) enabled nickel-catalyzed Kumada-Corriu cross-couplings of various arylmethyl ethers at room temperature by challenging $\mathrm{C}-\mathrm{O}$ activation.

Key words $\mathrm{C}-\mathrm{O}$ activation, arylation, cross-coupling, secondary phosphine oxide, nickel

Transition-metal-catalyzed cross-coupling reactions have emerged as a uniquely powerful tool for the assembly of substituted biaryl motifs. ${ }^{1}$ Thus far, these cross-couplings have heavily relied on aryl halides as electrophilic coupling reagents. In contrast, easily accessible phenol-based electrophiles have recently undergone a renaissance as attractive alternatives. ${ }^{2}$ On the basis of Wenkert's early studies from $1979,{ }^{3}$ the considerable potential of phenol-derived substrates has only recently been fully recognized. Thus, versatile cross-couplings have been realized with challenging carbamates, carbonates, sulfamates, silyloxyarenes, esters and ethers, among others, prominently featuring nickel catalysis. ${ }^{4}$ Generally, these nickel catalysts largely require electron-rich tertiary phosphines as stabilizing ligands to guarantee efficacy in the key $\mathrm{C}-\mathrm{O}$ bond scission. ${ }^{4}$ Unfortunately, these electron-rich tertiary phosphines are usually highly air-sensitive, with a documented half-life for the aerobic oxidation of tri-t-butyl-phosphine of a few minutes. ${ }^{5}$

The (heteroatom-substituted) secondary phosphine oxides (HA)SPOs represent uniquely powerful ancillary preligands for metal catalysis because of their unique features, including the air- and moisture-stable nature, among oth- ers. ${ }^{6}$ Notably, air-stable SPOs undergo a self-assembly process in the presence of transition metals to generate a monoanionic bidentate chelate coordination environment (Scheme 1, a). ${ }^{6}$ While Ackermann and others have unraveled the considerable potential of SPO complexes towards a wealth of efficient cross-coupling reactions with various aryl halides, ${ }^{7}$ the possibility of employing air-stable SPO preligands for more challenging $\mathrm{C}-\mathrm{O}$ activations with aryl ethers has thus far proven elusive. Within our program on sustainable transition-metal-catalyzed transformations ${ }^{8}$ and selective $\mathrm{C}-\mathrm{O}$ activation, ${ }^{9}$ we hence became attracted to probing the unprecedented use of air-stable SPOs preligands for cross-couplings with easily available aryl ethers, the result of which we report herein. Notable features of our findings include (i) air- and moisture-stable SPOs for efficient $\mathrm{C}-\mathrm{O}$ activations, (ii) earth-abundant nickel catalysis, and (iii) exceedingly mild reaction conditions at room temperature (Scheme 1, b).

(a)

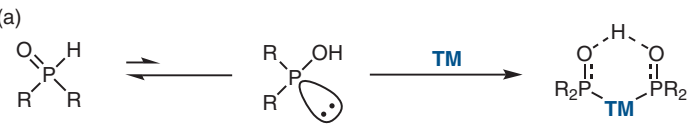

(b)

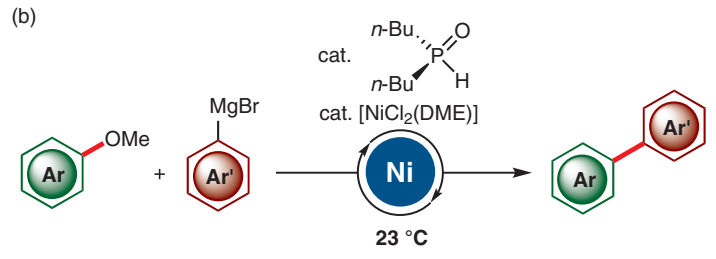

C- $O$ activation Earth-abundant nickel catalysis air- and moisture-stable SPO room temperature

Scheme 1 (a) Self-assembly with SPOs, (b) nickel/SPO-catalyzed C-O activation 
We initiated our studies by probing reaction conditions for the envisioned cross-coupling of ether $\mathbf{1 a}$ with $\mathrm{Ni}(\mathrm{acac})_{2}$ and $\mathrm{Ph}_{2} \mathrm{P}(\mathrm{O}) \mathrm{H}(\mathbf{L 1})$ in toluene at a room temperature of $23^{\circ} \mathrm{C}$ (Table 1 , entry 1 ). Among a variety of preligands and solvents, the electron-rich HASPO $\mathbf{L 7}$ as well as ( $n$ $\mathrm{Bu})_{2} \mathrm{P}(\mathrm{O}) \mathrm{H}(\mathbf{L 8})$ and THF gave optimal results, respectively (entries 2-13). $\mathrm{NiCl}_{2}$ (DME) proved to be most effective (entries 14-17). It is noteworthy that under otherwise identical reaction conditions, the bidentate ligand dppp featured a significantly inferior performance (entry 18). A control experiment verified the essential role of the nickel catalyst (entry 19).

Table 1 Optimization of the Nickel/SPO-Catalyzed C-O Activation of Ether $1 \mathbf{a}^{\mathrm{a}}$
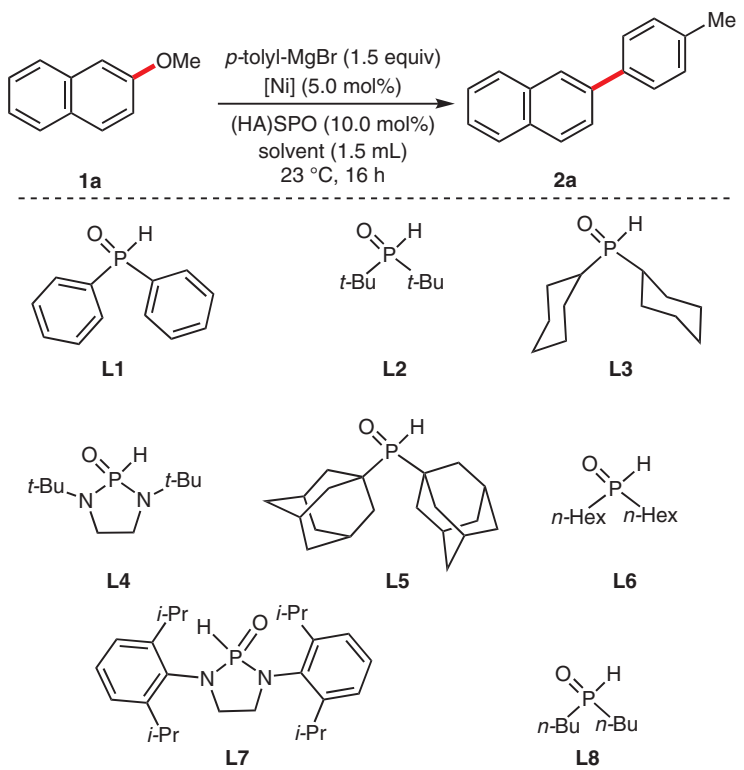

L6

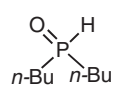

L8

\begin{tabular}{lllll}
\hline Entry & $\mathrm{Ni}$ Catalyst & SPO & Solvent & Yield (\%) \\
\hline 1 & $\mathrm{Ni}(\text { acac })_{2}$ & $\mathbf{L 1}$ & toluene & 10 \\
2 & $\mathrm{Ni}(\mathrm{acac})_{2}$ & $\mathbf{L 2}$ & toluene & 12 \\
3 & $\mathrm{Ni}(\text { acac })_{2}$ & $\mathbf{L 3}$ & toluene & 25 \\
4 & $\mathrm{Ni}(\text { acac })_{2}$ & $\mathbf{L 4}$ & toluene & 35 \\
5 & $\mathrm{Ni}(\text { acac })_{2}$ & $\mathbf{L 5}$ & toluene & 23 \\
6 & $\mathrm{Ni}(\mathrm{acac})_{2}$ & $\mathbf{L 6}$ & toluene & 50 \\
7 & $\mathrm{Ni}(\mathrm{acac})_{2}$ & $\mathbf{L 6}$ & THF & 64 \\
8 & $\mathrm{Ni}(\text { acac })_{2}$ & $\mathbf{L 1}$ & THF & 15 \\
9 & $\mathrm{Ni}(\text { acac })_{2}$ & $\mathbf{L 5}$ & THF & 21 \\
10 & $\mathrm{Ni}(\text { acac })_{2}$ & $\mathbf{L 3}$ & THF & 60 \\
11 & $\mathrm{Ni}(\text { acac })_{2}$ & $\mathbf{L 4}$ & THF & 48 \\
12 & $\mathrm{Ni}(\text { acac })_{2}$ & $\mathbf{L 7}$ & THF & 69 \\
13 & $\mathrm{Ni}(\text { acac })_{2}$ & $\mathbf{L 8}$ & THF & 83 \\
14 & $\mathrm{Ni}(\mathrm{OTf})_{2}$ & $\mathbf{L 8}$ & THF & 53 \\
15 & $\mathrm{NiBr} 2$ & $\mathbf{L 8}$ & THF & $\mathrm{n} . \mathrm{r}$. \\
& & & &
\end{tabular}

\begin{tabular}{ccccc}
\hline Entry & $\mathrm{NiCatalyst}$ & $\mathrm{SPO}$ & Solvent & Yield (\%) \\
\hline $\mathbf{1 6}$ & $\mathrm{NiCl}_{2}$ (DME) & $\mathbf{L 8}$ & THF & $\mathbf{9 0}$ \\
17 & $\mathrm{NiCl}_{2}$ (DME) & $\mathbf{L 8}$ & $\mathrm{THF}$ & $68^{\mathrm{b}}$ \\
18 & $\mathrm{NiCl}_{2}$ (DME) & dppp & THF & $39^{\mathrm{c}}$ \\
19 & - & $\mathbf{L 8}$ & $\mathrm{THF}$ & n.r. \\
\hline
\end{tabular}

a Reaction conditions: $1 \mathrm{a}(0.50 \mathrm{mmol}), \mathrm{p}-\mathrm{Tol} \mathrm{MgBr}(0.75 \mathrm{mmol})$, [Ni] $(5.0$ mol\%), (HA)SPO (10 mol\%), solvent $(1.5 \mathrm{~mL}), 23^{\circ} \mathrm{C}, 16 \mathrm{~h}$; yield of isolated product given; n.r. = no reaction.

${ }^{b}$ SPO L8 (5.0 mol\%).

' $\operatorname{dppp}(5.0 \mathrm{~mol} \%)$

Having the optimized reaction conditions for the nickel/SPO-catalyzed C-O activation in hand, we tested its versatility with a representative set of ethers $\mathbf{1}$ (Scheme 2). Thus, a variety of naphthyl ethers $\mathbf{1}$ were identified as viable substrates for the Kumada-Corriu cross-coupling to deliver the desired products $\mathbf{2}$ with high catalytic efficacy. Notably, the nickel catalyst derived from the air-stable SPO L8 even proved amenable to the chemoselective synthesis of biaryl $\mathbf{2 b}$ and the sterically congested mesityl nucleophiles with comparable levels of activity ( $\mathbf{2 d}$ and $\mathbf{2 i}$ ).<smiles>COc1ccccc1-c1ccc(-c2ccc3ccccc3c2)cc1</smiles>

Scheme 2 Scope of SPO/nickel-catalyzed C-O activation; ${ }^{\text {a }}$ with $\mathrm{NiCl}_{2}$ (DME) (10 mol\%) and $\mathbf{L 8}$ (20 mol\%) 
Based on our previous literature reports, ${ }^{6 c-d, 10}$ the working mode of the air-stable SPO-enabled $\mathrm{C}-\mathrm{O}$ activation is suggested to initially involve the formation of complex $\mathbf{3}$ through self-assembly, along with the subsequent $\mathrm{C}-\mathrm{O}$ activation by the key hetero-bimetallic intermediate $\mathbf{4}$ (Scheme 3 ).

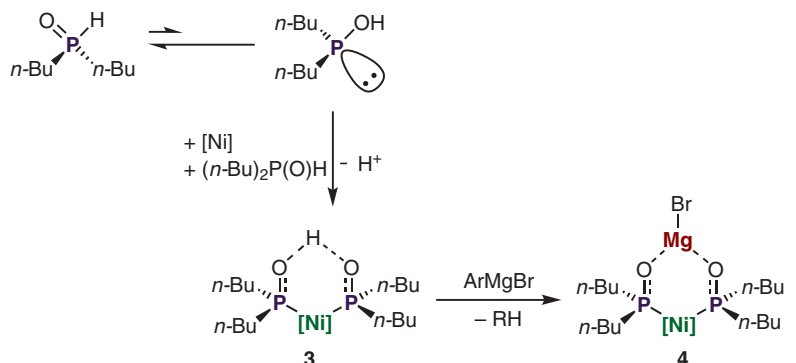

Scheme 3 Plausible working mode of SPOs for $\mathrm{C}-\mathrm{O}$ activation

In summary, we have reported on the first use of airstable secondary phosphine oxides (SPOs) for challenging cross-couplings of aryl ethers by $\mathrm{C}-\mathrm{O}$ activation. ${ }^{11}$ Thus, in situ generated nickel catalysts enabled efficient KumadaCorriu arylations of naphthyl ethers at room temperature, even when using sterically hindered aryl nucleophiles.

\section{Funding Information}

Generous support by the European Research Council under the European Community's Seventh Framework Program (FP7 20072013)/ERC Grant agreement no. 307535, and the Regione Lombardia - Cariplo Foundation is gratefully acknowledged.

\section{Supporting Information}

Supporting information for this article is available online at https://doi.org/10.1055/s-0037-1611663.

\section{References and Notes}

(1) (a) Cherney, A. H.; Kadunce, N. T.; Reisman, S. E. Chem. Rev. 2015, 115, 9587. (b) Johansson Seechurn, C. C.; Kitching, M. O.; Colacot, T. J.; Snieckus, V. Angew. Chem. Int. Ed. 2012, 51, 5062. (c) Modern Arylation Methods, 2nd ed; Ackermann, L., Ed.; Wiley-VCH: Weinheim, 2009. (d) Beller, M.; Bolm, C. Transition Metals for Organic Synthesis; Wiley-VCH: Weinheim, 2004. (e) Miyaura, N.; Suzuki, A. Chem. Rev. 1995, 95, 2457.

(2) Kozhushkov, S. I.; Potukuchi, H. K.; Ackermann, L. Catal. Sci. Technol. 2013, 3, 562.

(3) (a) Wenkert, E.; Michelotti, E. L.; Swindell, C. S.; Tingoli, M. J. Org. Chem. 1984, 49, 4894. (b) Wenkert, E.; Michelotti, E. L.; Swindell, C. S. J. Am. Chem. Soc. 1979, 101, 2246.

(4) Representative reviews: (a) Tobisu, M.; Chatani, N. Acc. Chem. Res. 2015, 48, 1717. (b) Su, B.; Cao, Z.-C.; Shi, Z.-J. Acc. Chem. Res. 2015, 48, 886. (c) Tollefson, E. J.; Hanna, L. E.; Jarvo, E. R. Acc. Chem. Res. 2015, 48, 2344. (d) Tasker, S. Z.; Standley, E. A.;
Jamison, T. F. Nature 2014, 509, 299. (e) Cornella, J.; Zarate, C.; Martin, R. Chem. Soc. Rev. 2014, 43, 8081. (f) Li, B. J.; Yu, D. G.; Sun, C. L.; Shi, Z. J. Chem. Eur. J. 2011, 17, 1728. (g) Rosen, B. M.; Quasdorf, K. W.; Wilson, D. A.; Zhang, N.; Resmerita, A.-M.; Garg, N. K.; Percec, V. Chem. Rev. 2011, 111, 1346. (h) Yu, D.-G.; Li, B.-J.; Shi, Z.-J. Acc. Chem. Res. 2010, 43, 1486. Selected examples: (i) Wang, T.-H.; Ambre, R.; Wang, Q.; Lee, W.-C.; Wang, P.-C.; Liu, Y.; Zhao, L.; Ong, T.-G. ACS Catal. 2018, 8, 11368. (j) Cao, Z.-C.; Luo, Q.-Y.; Shi, Z.-J. Org. Lett. 2016, 18, 5978. (k) Zhang, J.; Xu, J.; Xu, Y.; Sun, H.; Shen, Q.; Zhang, Y. Organometallics 2015, 34, 5792. (1) Iglesias, M. J.; Prieto, A.; Nicasio, M. C. Org. Lett. 2012, 14, 4318. (m) Xie, L.-G.; Wang, Z.-X. Chem. Eur. J. 2011, 17, 4972. (n) Dankwardt, J. W. Angew. Chem. Int. Ed. 2004, 43, 2428. For general reviews on nickel catalyzed transformations, see: (o) Castro, L. C. M.; Chatani, N. Chem. Lett. 2015, 44, 410. (p) Yamaguchi, J.; Muto, K.; Itami, K. Eur. J. Org. Chem. 2013, 19. (q) Nakao, Y. Chem. Rec. 2011, 11, 242, and references cited therein.

(5) Netherton, M. R.; Fu, G. C. Org. Lett. 2001, 3, 4295.

(6) Select reviews: (a) Herault, D.; Nguyen, D. H.; Nuel, D.; Buono, G. Chem. Soc. Rev. 2015, 44, 2508. (b) Shaikh, T. M.; Weng, C.-M.; Hong, F.-E. Coord. Chem. Rev. 2012, 256, 771. (c) Ackermann, L. Isr. J. Chem. 2010, 50, 652. (d) Ackermann, L. Synthesis 2006, 1557. (e) Dubrovina, N. V.; Börner, A. Angew. Chem. Int. Ed. 2004, 43, 5883.

(7) (a) Ghorai, D.; Müller, V.; Keil, H.; Stalke, D.; Zanoni, G.; Tkachenko, B. A.; Schreiner, P. R.; Ackermann, L. Adv. Synth. Catal. 2017, 359, 3137. (b) Hu, C.-Y.; Chen, Y.-Q.; Lin, G.-Y.; Huang, M.-K.; Chang, Y.-C.; Hong, F.-E. Eur. J. Inorg. Chem. 2016, 3131. (c) Cano, I.; Tschan, M. J. L.; Martínez-Prieto, L. M.; Philippot, K.; Chaudret, B.; van Leeuwen, P. W. N. M. Catal. Sci. Technol. 2016, 6, 3758. (d) Wellala, N. P.; Guan, H. Org. Biomol. Chem. 2015, 13, 10802. (e) Cano, I.; Huertos, M. A.; Chapman, A. M.; Buntkowsky, G.; Gutmann, T.; Groszewicz, P. B.; van Leeuwen, P. W. N. M. J. Am. Chem. Soc. 2015, 137, 7718. (f) Ackermann, L.; Kapdi, A. R.; Fenner, S.; Kornhaass, C.; Schulzke, C. Chem. Eur. J. 2011, 17, 2965. (g) Ackermann, L.; Potukuchi, H. K.; Kapdi, A. R.; Schulzke, C. Chem. Eur. J. 2010, 16, 3300. (h) Ackermann, L.; Vicente, R.; Hofmann, N. Org. Lett. 2010, 11, 4274. (i) Achard, T.; Giordano, L.; Tenaglia, A.; Gimbert, Y.; Buono, G. Organometallics 2010, 29, 3936. (j) Christiansen, A.; Selent, D.; Spannenberg, A.; Baumann, W.; Franke, R.; Börner, A. Organometallics 2010, 29, 3139. (k) Christiansen, A.; Li, C.; Garland, M.; Selent, D.; Ludwig, R.; Spannenberg, A.; Baumann, W.; Franke, R.; Börner, A. Eur. J. Org. Chem. 2010, 2733. (1) Ackermann, L.; Barfüßer, S. Synlett 2009, 808. (m) Yang, D. X.; Colletti, S. L.; Wu, K.; Song, M.; Li, G. Y.; Shen, H. C. Org. Lett. 2009, 11, 381. (n) Billingsley, K. L.; Buchwald, S. L. Angew. Chem., Int. Ed. Engl. 2008, 47, 4695. (o) Ackermann, L.; Born, R.; Spatz, J. H.; Meyer, D. Angew. Chem. Int. Ed. 2005, 44, 7216.

(8) (a) Gandeepan, P.; Müller, T.; Zell, D.; Cera, G.; Warratz, S.; Ackermann, L. Chem. Rev. 2019, in press; doi: 10 1021/acs.chemrev.8b00507. (b) Lorion, M. M.; Maindan, K.; Kapdi, A. R.; Ackermann, L. Chem. Soc. Rev. 2017, 46, 7399. (c) Moselage, M.; Li, J.; Ackermann, L. ACS Catal. 2016, 6, 498. (d) Liu, W.; Ackermann, L. ACS Catal. 2016, 6, 3743.

(9) (a) Sauermann, N.; Loup, J.; Kootz, D.; Berkessel, A.; Ackermann, L. Synthesis 2017, 49, 3476. (b) Song, W.; Ackermann, L. Angew. Chem. Int. Ed. 2012, 51, 8251. (c) Ackermann, L.; Pospech, J.; Potukuchi, H. K. Org. Lett. 2012, 14, 2146. (d) Ackermann, L.; 
Althammer, A.; Born, R. Angew. Chem. Int. Ed. 2006, 45, 2619. (e) Moselage, M.; Sauermann, N.; Richter, S. C.; Ackermann, L. Angew. Chem. Int. Ed. 2015, 54, 6352.

(10) Ackermann, L. Synlett 2007, 507.

(11) Representative Experimental Procedure and Characterization Data

A mixture of 2-methoxynaphthalene (1a) (79 $\mathrm{mg}, 0.5 \mathrm{mmol}$ ), [ $\mathrm{NiCl}_{2}$ (DME)] (6.0 mg, $\left.0.025 \mathrm{mmol}, 5.0 \mathrm{~mol} \%\right)$, and $\mathbf{~} \mathbf{8}(8.0 \mathrm{mg}$, $0.05 \mathrm{mmol}, 10.0 \mathrm{~mol} \%$ ) was stirred in THF $(1.5 \mathrm{~mL})$ for $2 \mathrm{~min}$ at ambient temperature under $\mathrm{N}_{2}$. Then, $p$ - $\mathrm{TolMgBr}(1.0 \mathrm{M}$ in THF, $0.75 \mathrm{~mL}, 0.75 \mathrm{mmol}$ ) was added, and the resulting solution was stirred for $16 \mathrm{~h}$ at ambient temperature. To the reaction was added aqueous $\mathrm{HCl}(1 \mathrm{M}, 5 \mathrm{~mL})$ and then EtOAc $(5 \mathrm{~mL})$, and the separated aqueous phase was extracted with EtOAc $(2 \times 5 \mathrm{~mL})$.
The combined organic layers were dried with anhydrous $\mathrm{Na}_{2} \mathrm{SO}_{4}$ and concentrated in vacuo. The remaining residue was purified by column chromatography on silica gel ( $n$-hexane) to yield $\mathbf{2 a}$ (98 mg, 90\%) as a colorless solid. Mp 93-95 ${ }^{\circ} \mathrm{C}$. IR (ATR): 3054, 3024, 1501, 1351, 893, 856, 811, $748 \mathrm{~cm}^{-1}$. ${ }^{1} \mathrm{H}$ NMR $(300 \mathrm{MHz}$, $\left.\mathrm{CDCl}_{3}\right): \delta=8.14(\mathrm{~d}, J=1.4 \mathrm{~Hz}, 1 \mathrm{H}), 8.03-7.93(\mathrm{~m}, 3 \mathrm{H}), 7.85(\mathrm{dd}$, $J=8.5,1.9 \mathrm{~Hz}, 1 \mathrm{H}), 7.74(\mathrm{~d}, J=8.1 \mathrm{~Hz}, 2 \mathrm{H}), 7.64-7.54(\mathrm{~m}, 2 \mathrm{H})$, $7.40(\mathrm{dd}, J=8.5,0.6 \mathrm{~Hz}, 2 \mathrm{H}), 2.53(\mathrm{~s}, 3 \mathrm{H}) .{ }^{13} \mathrm{C}$ NMR $(75 \mathrm{MHz}$, $\left.\mathrm{CDCl}_{3}\right): \delta=138.5\left(\mathrm{C}_{\mathrm{q}}\right), 138.3\left(\mathrm{C}_{\mathrm{q}}\right), 137.2\left(\mathrm{C}_{\mathrm{q}}\right), 133.8\left(\mathrm{C}_{\mathrm{q}}\right), 132.5$ $\left(\mathrm{C}_{\mathrm{q}}\right), 129.6(\mathrm{CH}), 128.4(\mathrm{CH}), 128.2(\mathrm{CH}), 127.7(\mathrm{CH}), 127.3(\mathrm{CH})$, 126.3 (CH), $125.8(\mathrm{CH}), 125.6(\mathrm{CH}), 125.5(\mathrm{CH}), 21.2\left(\mathrm{CH}_{3}\right) . \mathrm{MS}$ (EI): $m / z$ (relative intensity) $=218[\mathrm{M}]^{+}(100), 217(41), 202(35)$. HRMS (EI): $\mathrm{m} / z[\mathrm{M}]^{+}$calcd for $\left[\mathrm{C}_{17} \mathrm{H}_{14}\right]^{+}:$218.1096; found: 218.1094. 